BULL. AUSTRAL. MATH. SOC.

VOL. 27 (1983), 285-305.

\title{
ON LINEAR OPERATORS ON ORDERED BANACH SPACES
}

\author{
SADAYUKI YamamURO
}

\begin{abstract}
The ordex structure of the space of all continuous linear operators on an ordered Banach space is studied. The main topic is the Robinson property, that is, the norm of a positive linear operator is attained on the positive unit cone.
\end{abstract}

Let $B$ be a real Banach space ordered by a closed and proper cone $B_{+}$. The norm of $B$ is said to be monotone if $0 \leq a \leq b$ implies $\|a\| \leq\|b\|$, absolutely monotone if $-b \leq a \leq b$ implies $\|a\| \leq\|b\|$, and a Riesz norm if both the norm and its dual norm are absolutely monotone, where the dual $B^{*}$ of $B$ is ordered by the dual cone $B_{+}^{*}$ defined by

$$
B_{+}^{*}=\left\{f \in B^{*}: f(a) \geq 0 \text { for every } a \geq 0\right\} \text {. }
$$

The cone $B_{+}^{*}$ is proper if and only if $B_{+}$is quasi-generating; that is, $\overline{B_{+}-B_{+}}=B$. Throughout this paper $B_{+}$is always assumed to be quasigenerating.

Let $L(B)$ be the space of all continuous linear operators of $B$ into itself. The norm on $L(B)$ is defined by

$$
\|u\|=\sup \left\{\|u(a)\|: a \in B_{1}\right\} \text { for } u \in L(B),
$$

where $B_{1}$ is the closed unit ball of $B$. Then $L(B)$ is a Banach space

Received 19 November 1982. This work has been done while the author stayed at the Mathematics Research Center, University of Wisconsin. He thanks the director, Professor John Nohel, for his hospitality. The author is indebted to Professor D.W. Robinson for many helpful communications. Some of the results in this paper have been obtained independently by him and Dr C.J.K. Batty. 
and it is ordered by the cone

$$
L(B)_{+}=\{u \in L(B): u(a) \geq 0 \text { for every } a \geq 0\},
$$

which is closed and proper.

These notions have played essential rôles in a theory developed by Robinson [4, 5]. He has shown, in particular, that the norm

$$
\|u\|_{+}=\sup \{\|u(a)\|: a \in P\} \text { for } u \in L(B) \text {, }
$$

where $P=B_{1} \cap B_{+}$, fits into the theory better than the norm of $L(B)$ and the equality

$$
\|u\|_{+}=\|u\| \text { for every } u \in L(B)_{+}
$$

holds if the norm of $B$ is a Riesz norm.

This equality is expressed in terms of the norm and order structure of the ordered Banach space $L(B)$ and, therefore, better understanding of this equality requires further investigations on the order structure of $L(B)$.

To carry out this investigation, we shall use the notion of the halfnorm which has been introduced by Arendt, Chernoff and Kato [1]. As stated above, $B$ is a real Banach space ordered by a closed and proper cone $B_{+}$. Then a half norm on $B$ is a real-valued function $N$ on $B$ such that the following conditions are satisfied:

(1) there exists a constant $\alpha \geq 0$ such that $N(a) \leq \alpha\|a\|$ for all $a \in B$;

(2) $N(a+b) \leq N(a)+N(b)$ for $a, b \in B$;

(3) $N(\lambda a)=\lambda N(\alpha)$ for $\lambda \geq 0$ and $a \in B$;

(4) $N(a)+N(-a)=0$ implies $a=0$.

In particular,

$$
N(a)=\inf \left\{\|a+b\|: b \in B_{+}\right\}
$$

is a half-norm on $B$ and $0 \leq N(a) \leq\|a\|$. This is called the canonical half-norm of $B_{+}$. Unless otherwise stated, we shall always denote by $N$ the canonical half-norm of $B_{+}$.

It has been shown in [9] that 


$$
N(a)=\sup \left\{f(a): f \in P^{*}\right\} \text { for all } a \in B,
$$

where $P^{*}=B_{1}^{*} \cap B_{+}^{*}$ and $B_{1}^{*}$ denotes the closed unit ball of $B^{*}$. Since $B_{+}^{*}$ is proper, we can define the canonical half-norm of $B_{+}^{*}$ :

$$
N(f)=\inf \left\{\|f+g\|: g \in B_{+}^{*}\right\} .
$$

It has been proved in [9] that

$$
N(f)=\sup \{f(a): a \in P\} \text { for all } f \in B^{*} .
$$

We shall frequently use a simple fact that the norm of $B$ is monotone if and only if $\|a\|=N(a)$ for every $a \in B_{+}$.

\section{Order relations in $L(B)$}

An element $u$ of $L(B)$ is said to be positive if $u(a) \geq 0$ for every positive element $a$ of $B$. The set of all positive elements of $L(B)$ is denoted by $L(B)_{+}$and the closed unit ball of $L(B)$ is denoted by $L(B)_{1}$. We set

$$
\mathbf{P}=L(B)_{1} \cap L(B)_{+}
$$

The dual $L(B) *$ of $L(B)$ is then equipped with the order structure defined by $L(B)_{+}$. We denote the set of all positive elements of $L(B)^{*}$ by $L(B)_{+}^{*}$ and the closed unit ball of $L(B)^{*}$ by $L(B)_{1}^{*}$. We set

$$
\mathbb{P}^{*}=L(B)_{1}^{*} \cap L(B)_{+}^{*}
$$

Since $B$ is assumed to be quasi-generating, $L(B)+$ is a proper and closed cone in $L(B)$. Hence the canonical half-norm

$$
N(u)=\inf \{\|u+v\|: v \geq 0\}
$$

is defined for all $u \in L(B)$, and, as we have shown in [9], it has another expression

$$
N(u)=\sup \left\{F(u): F \in \mathbf{P}^{*}\right\}
$$

We define the second half-norm $\bar{N}$ on $L(B)$ by

$$
\bar{N}(u)=\sup \{N(u(a)): a \in P\} \text {. }
$$

Then, since

$$
N(u(a)) \leq N(u(a)+v(a)) \leq\|u+v\|
$$


for all $a \in P$ and $v \in L(B)_{+}$, we have

$$
\bar{N}(u) \leq N(u) \leq\|u\| \text { for all } u \in L(B) \text {. }
$$

Relations among these quantities will be studied in the next section. Here we shall give some general remarks on the order structure of $L(B)$.

First, we note that $L(B)_{+}$is not necessarily generating even when $B$ is a Banach lattice. It is generating if and only if every element of $L(B)$ is "regular" in the sense of Kantorovitch [3].

Secondly, we can consider $N\left(u^{*}\right)$ for the dual $u^{*}$ of $u \in L(B)$ only when $B_{+}^{*}$ is quasi-generating. When $B_{+}^{*}$ is quasi-generating, $N\left(u^{*}\right) \leq N(u)$ is the best we can have unless $B$ is reflexive. However, the second half-norm presents no such difficulties.

(1.1). $\bar{N}(u)=\bar{N}\left(u^{*}\right)$ for every $u \in L(B)$.

Proof. The definition of $\bar{N}\left(u^{*}\right)$,

$$
\bar{N}\left(u^{*}\right)=\sup \left\{N\left(u^{*}(f)\right): f \in P^{*}\right\},
$$

is always meaningful and

$$
\begin{aligned}
\bar{N}\left(u^{*}\right) & =\sup \left\{u^{*}(f)(a): a \in P, f \in P^{*}\right\} \\
& =\sup \left\{f(u(a)): a \in P, f \in P^{*}\right\} \\
& =\sup \{N(u(a)\}: a \in P\}=\bar{N}(u) .
\end{aligned}
$$

We recall that the norm of $L(B)$ is monotone if and only if $\|u\|=N(u)$ for every $u \in L(B)_{+}$.

(1.2). If the norm of $L(B)$ is monotone, the norms of $B$ and $B^{*}$ are both monotone.

Proof. For $a \in B$ and $f \in B^{*}$, we define an element $a \otimes f$ of $L(B)$ by

$$
(a \otimes f)(x)=f(x) a \text { for all } x \in B .
$$

Then $\|a \otimes f\|=\|a\|\|f\|$. Now suppose $0 \leq a \leq b$ and $f \in P^{*}$. Then $0 \leq a \otimes f \leq b \otimes f$ and, hence,

$$
\|a\|\|f\|=\|a \otimes f\| \leq\|b \otimes f\|=\|b\|\|f\| .
$$

Therefore the norm of $B$ is monotone. Similarly, it can be proved that the norm of $B^{*}$ is monotone.

The converse of (1.2) is not true. In contrast to this situation, the 
absolute monotonicity is a symmetric property in the following sense.

(1.3). The following conditions are equivalent:

(1) the norm of $L(B)$ is absolutely monotone;

(2) the norms of $B$ and $B^{*}$ are both absolutely monotone, that is, the norm of $B$ is a Riesz norm.

Proof. (I) $\Rightarrow(2)$. Suppose that $-b \leq a \leq b$ and $f \in P^{*}$. Then $-b \otimes f \leq a \otimes f \leq b \otimes f$ and we have $\|a\| \leq\|b\|$ by the same argument as in (1.2). Similarly, the norm of $B^{*}$ is absolutely monotone.

(2) $\Rightarrow(1)$. Suppose that $-v \leq u \leq v$ and $a \in B_{1}$. Then, for any $\varepsilon>0$, there exists $b \geq 0$ such that $-b \leq a \leq b$ and $\|b\| \leq(1+\varepsilon)\|a\|$ (see [9]). Then

$$
\pm u(a)= \pm u\left(\frac{b+a}{2}\right) \mp u\left(\frac{b-a}{2}\right) \leq v\left(\frac{b+a}{2}\right)+v\left(\frac{b-a}{2}\right)=v(b),
$$

and, therefore,

$$
\|u(a)\| \leq\|v(b)\| \leq(1+\varepsilon)\|v\|\|a\|
$$

Hence $\|u\| \leq\|v\|$.

There is another characterization of Fiesz norms which uses the notion of orthogonal generation. We say that $B_{+}$is orthogonally generating (see [6]) if every element $a$ of $B$ has a decomposition $a=a_{+}-a_{-}$such that $a_{ \pm} \geq 0$ and $\|a\|=\left\|a_{+}+a_{-}\right\|$. Robinson [4] has observed that, if the norm of $B$ is a Riesz norm, the dual cone $B_{+}^{*}$ is orthogonally generating. This observation turns out to be an essential one as the following result shows. We note that $u \leq v$ in $L(B)$ if and only if $u^{*} \leq v^{*}$ for the duals $u^{*}$ and $v^{*}$ of $u$ and $v$ respectively.

(1.4). The following conditions are equivalent:

(1) the norm of $B$ is a Riesz norm;

(2) $B_{+}^{*}$ and $B_{+}^{* *}$ are orthogonally generating.

Proof. (1) $\Rightarrow(2)$. When the norm of $B$ is a Riesz norm, the norm of $B^{*}$ is also a Riesz norm. Hence (2) follows from Robinson's result cited above.

$(2) \Rightarrow(1)$. We shall prove that the norm of $L(B)$ is absolutely monotone. Then (1.3) will imply that the norm of $B$ is a Riesz norm. Now 
suppose that $\pm u \leq v$ in $L(B)$. Let $a \in B_{1}$ and $f \in B_{1}^{*}$. We denote by $\hat{a}$ the image of $a$ by the imbedding of $B$ into $B^{* *}$, and let $\hat{a}=\hat{a}_{+}=\hat{a}_{=}$and $f=f_{+}-f_{-}$be orthogonal decompositions. Then

$$
\begin{aligned}
f(u(a)) & =\hat{a}\left(u^{*}(f)\right) \\
& =\hat{a}_{+}\left(u^{*}\left(f_{+}\right)\right)+\hat{a}_{-}\left(u^{*}\left(f_{-}\right)\right)+\hat{a}_{+}\left(-u^{*}\left(f_{-}\right)\right)+\hat{a}_{-}\left(-u^{*}\left(f_{+}\right)\right) \\
& \leq \hat{a}_{+}\left(v^{*}\left(f_{+}\right)\right)+\hat{a}_{-}\left(v^{*}\left(f_{-}\right)\right)+\hat{a}_{+}\left(v^{*}\left(f_{-}\right)\right)+\hat{a}_{-}\left(v^{*}\left(f_{+}\right)\right) \\
& =\left(\hat{a}_{+}+\hat{a}_{-}\right) v^{*}\left(f_{+}+f_{-}\right) \\
& \leq\left\|a_{+}^{+}\right\| a_{-}\left\|v^{*}\right\|\left\|_{+}+f_{-}\right\|=\|a\|\|v\|\|f\| .
\end{aligned}
$$

Hence $\|u\| \leq\|v\|$.

It follows from (1.4) that, if $B_{+}$and $B_{+}^{*}$ are orthogonally generating (then the norm of $B$ is a Riesz norm), then $B_{+}^{* *}$ is also orthogonally generating.

Dr C.J.K. Batty has shown that (1.4) follows from the fact that $B_{+}$ is absolutely dominated (see [4]) if and only if it is orthogonally generating.

\section{The norm and the half-norms}

For $a \in B$ and $f \in B^{*}$, we define an element of $L(B)^{*}$ by

$$
(a, f)(u)=f(u(a)) \text { for all } u \in L(B) \text {. }
$$

For subsets $X \subset B$ and $Y \subset B^{*}$, we set

$$
(X, Y)=\{(a, f): a \in X \text { and } f \in Y\} \text {. }
$$

For a subset $Z \subset L(B)^{*}$, we define the polars by

$$
Z^{\circ}=\{u \in L(B): F(u) \leq 1 \text { for every } F \in Z\}
$$

and

$$
Z^{\circ \circ}=\left\{F \in L(B)^{*}: F(u) \leq 1 \text { for every } u \in Z^{\circ}\right\} .
$$

The positive bipolars are defined by

$$
Z^{+}=Z^{\circ} \cap L(B)_{+}, Z^{0^{+}}=Z^{\circ 0} \cap L(B)_{+}^{*} \text { and } Z^{++}=Z^{+} \circ \cap L(B)_{+}^{*} \text {. }
$$

Then the following relation is obvious.

(2.1). $L(B)_{1}=\left(B_{1}, B_{1}^{*}\right)^{\circ}$.

Another relation that always holds is the following. 
(2.2). $\mathbf{P}^{*}=\left(B_{1}, B_{1}^{*}\right)^{0^{+}}$

This relation implies that $\mathbf{P}^{*}$ is contained in the convex $w^{*}$-closure $\left(B_{1}, B_{1}^{*}\right)^{\circ 0}$ of $\left(B_{1}, B_{1}^{*}\right)$.

We now compare two half-norms and the norm on $L(B)$. We recall that

$$
\begin{aligned}
& \bar{N}(u)=\sup \left\{(a, f)(u):(a, f) \in\left(P, P^{*}\right)\right\}, \\
& N(u)=\sup \left\{F(u): F \in \mathbb{P}^{*}\right\}
\end{aligned}
$$

and

$$
\|u\|=\sup \left\{(a, f)(u):(a, f) \in\left(B_{1}, B_{1}^{*}\right)\right\}
$$

(2.3). The following conditions are equivalent:

(1) $N(u)=\|u\|$ for all $u \in L(B)_{+}$;

(2) $\mathbf{P}=\left(\mathbf{P}^{*}\right)^{+}$;

(3) $\mathbf{P}$ is hereditary;

(4) the norm of $L(B)$ is monotone.

Proof. (1) $\Rightarrow(2)$. Since $P \subset\left(\mathbb{P}^{*}\right)^{+}$is obvious, suppose that $u \in\left(\mathbb{P}^{*}\right)^{+}$. Then $u \geq 0$ and $N(u) \leq 1$ and, hence, $\|u\| \leq 1$. Therefore $u \in P$.

$(2) \Rightarrow(3)$. Since $\left(P^{*}\right)^{+}$is obviously hereditary, so is $P$.

(3) $\Rightarrow(4)$. If $0 \leq u \leq v$, then $0 \leq\|v\|^{-1} u=\|v\|^{-1} v$ and $\|v\|^{-1} v \in \mathbf{P}$. Hence $\|v\|^{-1} u \in \mathbb{P}$ or $\|u\| \leq\|v\|$.

$(4) \Rightarrow(1)$. This has been proved in [9], Theorem 2.3.

(2.4). The following conditions are equivalent:

(1) $\bar{N}(u)=N(u)$ for every $u \in L(B)_{+}$;

(2) $\mathbf{P}^{*} \subset\left(P, P^{*}\right)^{++}$.

Proof. The equality ( 1 ) is equivalent to

$$
\left(P, P^{*}\right)^{+}=\left(\mathbf{P}^{*}\right)^{+} \text {. }
$$

Then $\mathbf{P}^{*} \subset\left(\mathbf{P}^{*}\right)^{++}=\left(P, P^{*}\right)^{++}$. Conversely, if condition (2) holds and $\bar{N}(u) \leq 1$ for some $u \in L(B)_{+}$, then 


$$
u \in\left(P, P^{*}\right)^{+} \subset\left(P, P^{*}\right)^{+++} \subset\left(\mathbb{P}^{*}\right)^{+}
$$

and hence, $N(u) \leq 1$. Therefore we have the equality (1).

Combining (2.3) and (2.4), we have the following.

(2.5). The following conditions are equivalent:

(1) $\bar{N}(u)=\|u\|$ for azz $u \in L(B)_{+}$

(2) $\mathbb{P}=\left(P, P^{*}\right)^{+}$.

In relation to condition (2) in (2.4), we note that the equality $\mathbb{P}^{*}=\left(P, P^{*}\right)^{++}$does not hold even when $B$ is a Banach lattice. In fact, if the equality holds, $P^{*}$ is hereditary and, hence, the norm of $L(B)^{*}$ is monotone. This then implies that $L(B)+$ is generating.

\section{The Robinson property}

We define the Robinson norm $\|u\|_{+}$for $u \in L(B)$ by

$$
\|u\|_{+}=\sup \{\|u(a)\|: a \in P\} .
$$

This norm has been introduced by Robinson [4] and has been shown to play an essential role in the theory of positive semigroups on ordered Banach spaces. We shall say that (the norm of) $B$ has the Robinson property if

$$
\|u\|_{+}=\|u\| \text { for all } u \in L(B)_{+} \text {. }
$$

Robinson [4] has noted that every Banach lattice, the self-adjoint part of every $C^{*}$-algebra and the predual of every $W^{*}$-algebra have the Robinson property. In fact, he has shown that the norm has the Robinson property if it is a Riesz norm.

Since

$$
\|u\|_{+}=\sup \left\{(a, f)(u):(a, f) \in\left(P, B_{1}^{*}\right)\right\}
$$

the following statement is obvious.

(3.1). The norm of $B$ has the Robinson property if and only if $\mathbf{P}=\left(p, B_{1}^{*}\right)^{+}$.

Note that the equality $\mathbf{P}=\left(P, B_{1}^{*}\right)^{+}$does not imply the monotonicity of the norm of $L(B)$, which is equivalent to that $\mathbf{P}$ is hereditary, because $\left(P, B_{1}^{*}\right)$ is not contained in $L(B)_{+}^{*}$. The monotonicity of the 
Robinson norm has simpler character.

(3.2). The following conditions are equivalent:

(1) the Robinson norm is monotone;

(2) the norm of $B$ is monotone;

(3) $\bar{N}(u)=\|u\|_{+}$for alz $u \in L(B)_{+}$.

Proof. (1) $\Rightarrow(2)$. We first note that $\|a \otimes f\|_{+}=N(f)\|a\|$ for $a \in B$ and $f \in B_{+}^{*}$. Since $0 \leq a \leq b$ and $f \geq 0$ imply $0 \leq a \otimes f \leq b \otimes f$, we have $\|a\| \leq\|b\|$.

(2) $\Rightarrow(3)$. Since $N(a)=\|a\|$ for every $a \in B_{+}$, we have $\|u(a)\|=N(u(a))$ for $u \geq 0$ and $a \geq 0$. This implies $\bar{N}(u)=\|u\|_{+}$for $u \geq 0$. Since $\bar{N}$ is monotone, it is evident that (3) implies (1).

We shall say that (the norm of) $B$ has the Robinson* property if

$$
\left\|u^{*}\right\|_{+}=\left\|u^{*}\right\| \text { for every } u \in L(B)_{+} \text {. }
$$

Since $\left\|u^{*}\right\|=\|u\|$, this is equivalent to $\left\|u^{*}\right\|_{+}=\|u\|$ for every $u \in L(B)_{+} \cdot$

(3.3). (1) If the norm of $B$ has the Robinson property, the dual norm is monotone.

(2) If the norm of $B$ has the Robinson* property, then it is monotone.

Proof. (1) follows from $\|a \otimes f\|_{+}=N(f)\|a\|$ for $f \geq 0$, and (2) follows from $\left\|(a \otimes f)^{*}\right\|_{+}=\|f\| N(a)$ for $a \geq 0$.

Now, if the norm of $B$ is monotone, we have, by (3.2), the following relation:

$$
\bar{N}(u)=\|u\|_{+} \leq N(u) \leq\|u\| \text { for } u \in L(B)_{+} .
$$

If we recall that $N(u)=\|u\|$ for all $u \in L(B)+$ if and only if the norm of $L(B)$ is monotone, the following statement follows immediately from (2.4) and (3.3).

(3.4). The following conditions are equivalent:

(1) the norm of $B$ is monotone and has the Robinson property; 
(2) the norm of $L(B)$ is monotone and $P^{*} \subset\left(P, P^{*}\right)^{++}$

(3) the norm of $B$ has the Robinson and Robinson * property.

Since Riesz norms are monotone, the norm of $B$ has the Robinson and Robinson* properties if it is a Riesz norm.

A norm on $B$ is called an order norm if

$$
\|a\|=N(a) \vee N(-a) \text { for all } a \in B \text {. }
$$

Robinson [5] has proved that the norm of $B$ has the Robinson property if it is an order norm and the dual norm is monotone. The converse is in fact true and it follows immediately from (3.3) (1).

(3.5). Let the norm of $B$ be an order norm. Then it has the Robinson property if and only if the dual norm is monotone.

We shall have a more general version of this result in $\$ 7$.

\section{The Robinson property in the duals}

Throughout this section, we assume that $B_{+}$is quasi-generating and normal, so that $B_{+}$is generating. Then the positive cone $L\left(B^{*}\right)_{+}$of $L\left(B^{*}\right)$ is proper under the canonical ordering. The second dual $B^{* *}$ of $B$ is also ordered canonically. The positive unit cone of $B^{* *}$ is denoted by $P^{* *}$. For $f \in P^{*}$ and $\xi \in P^{* *}$, we set

$$
(f, \xi)(V)=\xi(V(f)) \text { for every } V \in L\left(B^{*}\right)
$$

and

$$
\left(P^{*}, P^{* *}\right)=\left\{(f, \xi): f \in P^{*}, \xi \in P^{* *}\right\} .
$$

The the positive polar $\left(P^{*}, P^{* *}\right)^{+}$is, by definition,

$$
\left(P^{*}, P^{* *}\right)^{+}=\left\{V \geq 0:(f, \xi)(V) \leq 1 \text { for }(f, \xi) \in\left(P^{*}, P^{* *}\right)\right\} .
$$

The space $B$ is imbedded in $B^{* *}$ and, hence, $P$ is imbedded in $P^{* *}$. We denote the image of $P$ in $P^{* *}$ by this imbedding by the same $P$. Then we have the following relation.

(4.1). $\left(P^{*}, P\right)^{+}=\left(P^{*}, P^{* *}\right)^{+}$.

Proof. It is obvious that $\left(P^{*}, P\right)^{+} \supset\left(P^{*}, P^{*}\right)^{+}$. To prove the converse, suppose that $V \in\left(P^{*}, P\right)^{+}$. Then, for $f \in P^{*}$, we have $V(f) \in P^{\circ}$. It has been proved in [9] that $P^{\circ 0}=P^{* *}$ when the second 
polar is taken in $B^{* *}$. Hence $\xi(V(f)) \leq 1$ for every $\xi \in P^{* *}$, or $V \in\left(P^{*}, P^{*}\right)^{+}$.

Next we consider a correspondence between $L(B)$ and $L\left(B^{*}\right)$. For $u \in L(B)$, the dual $u^{*}$ belongs to $L\left(B^{*}\right)$ and it is $w^{*}-w^{*}$-continuous, that is, if $\left\{f_{\lambda}\right\}$ is a net in $B^{*}$ such that $f_{\lambda}(a) \rightarrow 0$ for every $a \in B$, then $\left\{u^{*}\left(f_{\lambda}\right)\right\}$ is a net in $B^{*}$ such that $u^{*}\left(f_{\lambda}\right)(a) \rightarrow 0$ for every $a \in B$. Conversely, if $V \in L\left(B^{*}\right)$ is $w^{*}-w^{*}$-continuous, we can find $V_{*} \in L(B)$ such that $\left\|V_{*}\right\|=\|V\|$ by

$$
V(f)(a)=f\left(V_{*}(a)\right) \text { for all }(a, f) \in\left(B, B^{*}\right) .
$$

Furthermore, $V_{*} \geq 0$ if and only if $V \geq 0$.

(4.2). The following conditions are equivalent:

(1) $\bar{N}(u)=\|u\|$ for every $u \in L(B)_{+}$

(2) $\bar{N}(V)=\|V\|$ for every positive and $w^{*}-w^{*}$-continuous element $V$ of $L\left(B^{*}\right)$.

Proof. $(1) \Rightarrow(2)$. Let $V$ be a positive and $w^{*}-w^{*}$-continuous element of $L\left(B^{*}\right)$. Then $V_{*} \in L(B)_{+}$. Hence, by the assumption, $\bar{N}\left(V_{*}\right)=\left\|V_{*}\right\|$. Now suppose that $V \in\left(P^{*}, P^{* *}\right)^{+}$. Then, by (4.1), $V \in\left(P^{*}, P\right)^{+}$and, hence, $V_{*} \in\left(P, P^{*}\right)^{+}$. Therefore $V_{*} \in \mathbb{P}$ by $(2.5)$, that is, $\left\|V_{*}\right\| \leq 1$. Then, again by (2.5), we have $\bar{N}(V)=\|V\|$.

$(2) \Rightarrow(1)$. For $u \in L(B)_{+}$we have $\bar{N}\left(u^{*}\right)=\left\|u^{*}\right\|$. Hence, by (1.1), we have $\bar{N}(u)=\|u\|$.

An immediate corollary is the following.

(4.3). If $\bar{N}(V)=\|v\|$ for every $V \in L\left(B^{*}\right)_{+}$, then $\bar{N}(u)=\|u\|$ for every $u \in L(B)_{+}$.

The following is another immediate corollary. In view of (3.3), the assumption that the norms of $B$ and $B^{*}$ are monotone is not restrictive.

(4.4). Suppose that the norms of $B$ and $B^{*}$ are montone.

(1) The norm of $B$ has the Robinson property if and only it has the Robinson * property.

(2) If the dual norm has the Robinson property, then the norm of $B$ has the Robinson property. 


\section{The N-decompositions}

As a preparation for obtaining another sufficient condition for the Robinson property, we shall introduce the notion of $N$-decomposability and give some of its basic properties.

When $B$ is a Banach lattice or the self-adjoint part of a $C^{*}-$ algebra, we always have

$$
N(a)=\left\|a_{+}\right\| \text {for every } a \in B,
$$

where $a_{+}$denotes the "positive part" of $a$. Hence, in these cases, every element has a decomposition $a=a_{+}-a_{-}$such that $a_{ \pm} \geq 0$, $N(a)=\left\|a_{+}\right\|$and $N(-a)=\left\|a_{-}\right\|$.

In general we call $B \quad N$-decomposable if every element of $B$ has an $N$-decomposition, that is, $a=a_{+}-a_{-}$with $a_{ \pm} \geq 0, N(a)=\left\|a_{+}\right\|$and $N(-a)=\left\|a_{-}\right\|$. An element may have more than one $N$-decomposition. However, a positive element $a \in B_{+}$has a unique $N$-decomposition $(a, 0)$.

If $B$ is $N$-decomposable, $B_{+}$is obviously generating and, furthermore, the norm of $B$ is monotone because $N(\alpha)=\|a\|$ for every $a \in B_{+}$. Hence $B_{+}^{*}$ is also generating.

(5.1). The following conditions are equivalent:

(1) $B^{*}$ is $N$-decomposable;

(2) for every $a \in B$ and $f \in B^{*}$,

$$
f(a) \leq N(f) N(a)+N(-f) N(-a) \text {. }
$$

Proof. (1) $\Rightarrow(2)$. Let $f=f_{+}-f_{-}$be an $N$-decomposition of $f \in B^{*}$. Then, for each $a \in B$,

$$
\begin{aligned}
f(a) & =f_{+}(a)+f_{-}(-a) \leq\left\|f_{+}\right\| N(a)+\left\|f_{-}\right\| N(-a) \\
& \leq N(f) N(a)+N(-f) N(-a) .
\end{aligned}
$$

(2) $\Rightarrow(1)$. Let $f \in B^{*}$ and set

$$
q(a)=N(f) N(a) \text { and } r(a)=f(a)+N(-f) N(a) .
$$

Then we have

$$
0 \leq q(a)+r(-a) \text { for all } a \in B \text {. }
$$

Therefore, by the double Hahn-Banach theorem [9], there exists $g \in B^{*}$ 
such that $g(a) \leq q(a)$ and $g(a) \leq r(a)$ for every $a \in B$. Then $f_{+}=g$ and $f_{-}=g-f$ supply an $N$-decomposition of $f$.

As the following result shows, $B^{*}$ is $N$-decomposable if $B$ is $N$-decomposable.

(5.2). The following conditions are equivalent:

(I) $B$ is N-decomposable;

(2) (i) $B^{*}$ is $N$-decomposable,

(ii) $\alpha P-\beta P$ is closed for every $\alpha>0$ and $\beta>0$.

Proof. $(1) \Rightarrow(2)$. Let $\left(a_{+}, a_{-}\right)$be an $N$-decomposition of $a \in B$. Then, for $f \in B^{*}$,

$$
\begin{aligned}
f(a) & =f\left(a_{+}\right)+(-f)\left(a_{-}\right) \leq N(f)\left\|a_{+}\right\|+N(-f)\left\|a_{-}\right\| \\
& \leq N(f) N(a)+N(-f) N(-a) .
\end{aligned}
$$

Therefore, by (5.1), $B^{*}$ is $N$-decomposable. Next, to prove (2) (ii), suppose that $a_{n}=\alpha b_{n}-\beta c_{n}, b_{n} \in P, c_{n} \in P$ and $a_{n} \rightarrow a$. Let $\left(a_{+}, a_{-}\right)$be an $N$-decomposition of $a$. Then, since

$$
\left\|a_{+}\right\|=N(a)=\lim _{n \rightarrow \infty} N\left(a_{n}\right), N\left(a_{n}\right) \leq N\left(\alpha b_{n}\right) \leq \alpha,
$$

and

$$
\left\|a_{-}\right\|=N(-a)=\lim _{n \rightarrow \infty} N\left(-a_{n}\right), N\left(-a_{n}\right) \leq N\left(\beta c_{n}\right)=\beta
$$

where have $a_{+} \in \alpha P$ and $a_{-} \in \beta P$. Hence $\alpha P-\beta P$ is closed.

$(2) \Rightarrow(1)$. Suppose that there exists $a \in B$ such that $a k N(a) P-N(-a) P$. Since $N(a) P-N(-a) P$ is closed and convex, there exists $f \in B^{*}$ such that

$$
\begin{aligned}
f(a) & >\sup \{f(x): x \in N(\alpha) P-N(-a) P\} \\
& =N(f) N(a)+N(-f) N(-a),
\end{aligned}
$$

which is a contradiction. Hence $a \in N(a) P-N(-a) P$ for every $a \in B$ and therefore $B$ is $N$-decomposable.

Condition (2) (ii) is satisfied by all Banach lattices and also the self-adjoint parts of $C^{*}$-algebras because they are $N$-decomposable. Furthermore, all the duals satisfy this condition because of $w^{*}$-compactness of $P^{*}$. Therefore 
(5.3). $B^{*}$ is $N$-decomposable if and only if $B^{* *}$ is $N$-decomposable.

An ordered Banach space $B$ will be called $N_{+}$-decomposable if every element $a \in B$ admits a decomposition $a=a_{+}-a_{-}$such that $a_{ \pm} \epsilon B_{+}$ and $N(a)=\left\|a_{+}\right\|$. We have proved in [9] that $B^{*}$ is $N_{+}$-decomposable if and only if the norm of $B$ is monotone.

(5.4). The following conditions are equivalent:

(1) $B$ is $N_{+}$-decomposable;

(2) (i) the norm of $B^{*}$ is monotone,

(ii) $P-B_{+}$is closed.

Proof. (1) $\Rightarrow(2)$. It has been proved in [9] that the norm of $B^{*}$ is monotone if and only if, for any $a \in B$ and $\varepsilon>0$, there exist $a_{ \pm} \in B_{+}$ such that $a=a_{+}-a_{-}$and $\left\|a_{+}\right\| \leq(1+\varepsilon)\|a\|$. When $B$ is $N_{+}$ decomposable, the condition is satisfied with $\varepsilon=0$. To prove that $P-B_{+}$is closed, suppose that $b_{n}-c_{n} \rightarrow a$ for $b_{n} \in P$ and $c_{n} \in B_{+}$. Let $\left(a_{+}, a_{-}\right)$be an $N_{+}$-decomposition of $a$. Then, since $N\left(a-b_{n}\right) \rightarrow 0$ and $N\left(b_{n}\right) \leq 1$, we have $N(a) \leq 1$, or equivalently, $a_{+} \in P$. Therefore $a \in P-B_{+}$.

$(2) \Rightarrow(1)$. The norm of $B^{*}$ is monotone if and only if

$$
P^{*}=B_{+}^{*} \cap\left(P^{*}-B_{+}^{*}\right) \text {. }
$$

The polar $\left(B_{+}^{*} \cap\left(P^{*}-B_{+}^{*}\right)\right)^{\circ}$ of the right-hand side coincides with $\overline{P-B_{+}}$, and

$$
\left(P^{*}\right)^{\circ}=B(N)=\{x \in B: N(x) \leq 1\} .
$$

Hence $B(N)=\overline{P-B_{+}}$and, since $P-B_{+}$is closed, we have

$$
B(N)=P-B_{+},
$$

which exactly means that $B$ is $N_{+}$-decomposable.

This, in particular, implies that the norm of $B^{*}$ is monotone if the norm of $B$ is monotone.

\section{Spaces of type $(N, p)$}

We set 


$$
\mu_{p}(a)=\left(N(a)^{p}+N(-a)^{p}\right)^{1 / p} \text { for } a \in B
$$

and

$$
\mu_{p}(f)=\left(N(f)^{p}+N(-f)^{p}\right)^{1 / p} \text { for } f \in B^{*} \text {, }
$$

where $1 \leq p \leq \infty$. We include the case when $p=\infty$ :

$$
\mu_{\infty}(a)=N(a) \vee N(-a) \text { for } a \in B
$$

and

$$
\mu_{\infty}(f)=N(f) \vee N(-f) \text { for } f \in B^{*} .
$$

Furthermore, we set

$$
\mu_{p}^{*}(f)=\sup \left\{f(a): \mu_{p}(a) \leq 1\right\} \text { for } f \in B^{*}
$$

and

$$
\mu_{p}^{\#}(a)=\sup \left\{f(a): \mu_{p}(f) \leq 1\right\} \text { for } a \in B \text {. }
$$

The following relations are obvious.

(6.1). (i) $\mu_{\infty}(a) \leq \mu_{p}(a) \leq \mu_{1}(a)$ for $a \in B$.

(ii) $\mu_{\infty}(f) \leq \mu_{p}(f) \leq \mu_{1}(f)$ for $f \in B^{*}$.

(iii) $\mu_{1}^{*}(f) \leq \mu_{p}^{*}(f) \leq \mu_{\infty}^{*}(f)$ for $f \in B^{*}$.

(iv) $\mu_{l}^{\#}(a) \leq \mu_{p}^{\#}(a) \leq \mu_{\infty}^{\#}(a)$ for $a \in B$.

(v) $\mu_{p}(a)=N(a)$ for $a \in B_{+} ; \mu_{p}(f)=N(f)$ for $f \in B_{+}^{*}$.

(vi) $\mu_{p}^{\#}(a) \leq\|a\|$ for $a \in B_{+} ; \mu_{p}^{*}(f) \leq\|f\|$ for $f \in B_{+}^{*}$.

Since $\mu_{\infty} \leq \mu_{p} \leq 2^{1 / p} \mu_{\infty}$ when $1 \leq p \leq \infty$, it is easy to see that

$\mu_{p}, \mu_{p}^{*}$ and $\mu_{p}^{\#}$ are all equivalent continuous norms on the spaces where they are defined. We also have the following relations.

(6.2). $\mu_{p}(a)=\sup \left\{f(a): \mu_{p}^{*}(f) \leq 1\right\}$ and

$$
\mu_{p}(f)=\sup \left\{f(a): \mu_{p}^{\#}(a) \leq 1\right\} \text {. }
$$


Proof. Since $\mu_{p}(a) \geq \sup \left\{f(a): \mu_{p}^{*}(f) \leq 1\right\}$ is obvious, we only need to construct $h \in B^{*}$ such that $\mu_{p}^{*}(h) \leq 1$ and $h(a)=\mu_{p}(a)$. For this purpose we take $f \in P^{*}$ and $g \in P^{*}$ such that $f(a)=N(a)$ and $g(-a)=N(-a)$ (see [6]). Now suppose that $p<\infty$ and set

$$
h=\mu_{p}(a)^{1-p}\left(N(a)^{p-1} f-N(-a)^{p-1} g\right) .
$$

Then $h(a)=\mu_{p}(a)$ and

$$
\begin{aligned}
\mu_{p}^{*}(h) & =\sup \left\{h(x): \mu_{p}(x) \leq 1\right\} \\
& \leq \mu_{p}(a)^{1-p} \sup \left\{N(a)^{p-1} N(x)+N(-a)^{p-1} N(-x): \mu_{p}(x) \leq 1\right\} \\
& =1 .
\end{aligned}
$$

When $p=\infty$, for the same $f$ and $g$ as above, we have $\mu_{\infty}^{*}(f) \leq 1$, $\mu_{\infty}^{*}(g) \leq 1$ and $\mu_{\infty}(a)=f(a) \vee g(a)$. Hence

$$
\mu_{\infty}(\alpha) \leq \sup \left\{f(\alpha): \mu_{p}^{*}(f) \leq 1\right\} \text {. }
$$

The second equality can be proved in the same manner.

We shall always denote $p /(p-1)$ by $q$. When $p=1$ or $p=\infty$, then $q=\infty$ or $q=1$ respectively.

(6.3). (1) For any $a_{ \pm} \in B_{+}$such that $a=a_{+}-a_{-}$,

$$
\mu_{p}(a) \leq \mu_{q}^{\#}(a) \leq\left(\left\|a_{+}\right\|^{p}+\left\|a_{-}\right\|^{p}\right)^{1 / p} \text {. }
$$

(2) For any $f_{ \pm} \in B_{+}^{*}$ such that $f=f_{+}-f_{-}$,

$$
\mu_{p}(f) \leq \mu_{q}^{*}(f) \leq\left(\left\|f_{+}\right\|^{p}+\left\|f_{-}\right\|^{p}\right)^{I / p} .
$$

Proof. Choose $f$ and $g$, and define $h$ in the same manner as in (6.2). Then $h(a)=\mu_{p}(a)$ and

$$
\mu_{q}(h)^{q}=N(h)^{q}+N(-h)^{q}=\mu_{p}(a)^{-p}\left(N(a)^{p}+N(-a)^{p}\right)=1 \text {. }
$$

Therefore $\mu_{p}(a) \leq \mu_{q}^{\#}(a)$.

Next let $a=a_{+}-a_{-}$and $a_{ \pm} \in B_{+}$. Then, for $f \in B^{*}$, 


$$
\begin{aligned}
f(a) & =f\left(a_{+}\right)+(-f)\left(a_{-}\right) \leq N(f)\left\|a_{+}\right\|+N(-f)\left\|a_{-}\right\| \\
& \leq \mu_{q}(f)\left(\left\|a_{+}\right\|^{p}+\left\|a_{-}\right\|^{p}\right)^{1 / p} .
\end{aligned}
$$

It then follows that $\mu_{q}^{\#}(a) \leq\left(\left\|a_{+}\right\|^{p}+\left\|a_{-}\right\|^{p}\right)^{1 / p}$.

When $p=\infty$ and $q=1$, the relation we need to prove is

$$
\mu_{\infty}(a) \leq \mu_{1}^{\#}(a) \leq\left\|a_{+}\right\| \vee\left\|a_{-}\right\| \text {. }
$$

To prove this we take $f \in P^{*}$ such that $f(a)=N(a)$. Then, since $\mu_{1}(f)=N(f) \leq 1$ by $(2.1)(v i)$, we conclude that $N(a) \leq \mu_{1}^{\#}(a)$.

Similarly, $N(-a) \leq \mu_{1}^{\#}(a)$, and, therefore, $\mu_{\infty}(a) \leq \mu_{1}^{\#}(a)$. The proof for $\mu_{1}^{\#}(a) \leq\left\|a_{+}\right\| \vee\left\|a_{-}\right\|$is the same as above. Thus (1) has been proved and

(2) can be proved similarly.

As a corollary, we have the following relations.

(6.4). (1) Suppose that $B$ is N-decomposable. Then

$$
\mu_{p}(a)=\mu_{q}^{\#}(a) \text { for all } a \in B \text {. }
$$

(2) Suppose that $B^{*}$ is $N$-decomposable. Then

$$
\mu_{p}(f)=\mu_{q}^{*}(f) \text { for azz } f \in B^{*}
$$

Proof. We only need to take $N$-decompositions $\left(a_{+}, a_{-}\right)$or $\left(f_{+}, f_{-}\right)$ in the right-hand sides of (2.3).

We say that $B$ is of type $(N, q)$ if $\|a\|=\mu_{p}(a)$ for all $a \in B$. Then (2.4) implies the following.

(6.5). (1) Suppose that $B$ is $N$-decomposable. If $B^{*}$ is of type $(N, q)$, then $B$ is of type $(N, p)$.

(2) Suppose that $B^{*}$ is $N$-decomposable. If $B$ is of type $(N, q)$, then $B^{*}$ is of type $(N, p)$.

Next we consider the relations between the spaces of type $(N, \infty)$ and of type $(N, 1)$. The following facts are fundamental.

(6.6). (1) $\|a\| \leq \mu_{1}(a)$ for every $a \in B$ if and only if $B_{1}^{*}$ is 
positively generated, that is, $B_{1}^{*} \subset P^{*}-P^{*}$.

(2) $\|f\| \leq \mu_{1}(f)$ for every $f \in B^{*}$ if and only if $B_{1}$ is positively quasi-generated, that is, $B_{1} \subset \overline{P-P}$.

Proof. Suppose that $\|a\| \leq \mu_{1}(a)$ for all $a \in B$. Then $B\left(\mu_{1}\right) \subset B_{1}$ where $B\left(\mu_{1}\right)=\left\{a \in B: \mu_{1}(\alpha) \leq 1\right\}$. Since we have $B\left(\mu_{1}\right)^{\circ}=P^{*}-P^{*}$ for the polar $B\left(\mu_{1}\right)^{\circ}$ of $B\left(\mu_{1}\right)$, we can conclude that $B_{1}^{*} \subset P^{*}-P^{*}$. Conversely, if $B_{1}^{*}$ is positively generated and $f \in B_{1}^{*}$, there exist $f_{ \pm} \in P^{*}$ such that $f=f_{+}-f_{-}$. Then

$$
f(a)=f_{+}(a)-f_{-}(a) \leq N(a)+N(-a)=\mu_{1}(a),
$$

and hence, $\|\alpha\| \leq \mu_{1}(a)$.

We can prove (2) in a similar manner by using the relation $B\left(\mu_{1}^{\#}\right)=\overline{P-P}$, where $B\left(\mu_{1}^{\#}\right)=\left\{a \in B: \mu_{1}^{\#}(a) \leq 1\right\}$.

We can now characterize those spaces of type $(N, \infty)$ whose duals are of type $(N, I)$.

(6.7). Let $B$ be of type $(N, \infty)$. Then the following conditions are equivalent:

(1) $B^{*}$ is of type $(N, 1)$;

(2) $B_{1}$ is positively quasi-generated;

(3) $B^{*}$ is $N$-decomposable.

Proof. $(I) \Rightarrow(2) . B^{*}$ is of type $(N, I)$ if and only if $\|f\|=\mu_{1}(f)$ for every $f \in B^{*}$. Hence (6.6) implies that $B_{1}$ is positively quasi-generated.

$(2) \Rightarrow(3)$. By the Grosberg-Krein theorem (see [2] and [6]), every $f \in B^{*}$ admits a Jordan decomposition

$$
f=f_{+}-f_{-}, f_{ \pm} \in B_{+}^{*} \text { and }\|f\|=\left\|f_{+}\right\|+\left\|f_{-}\right\| .
$$

It is obvious that $N(f) \leq\left\|f_{+}\right\|$and $N(-f) \leq\left\|f_{-}\right\|$. However, by (6.6), we have $\|f\| \leq N(f)+N(-f)$. Therefore $N(f)=\left\|f_{+}\right\|$and $N(-f)=\left\|f_{-}\right\|$. In 
other words, Jordan decompositions are $N$-decompositions when $B_{1}$ is positively quasi-generated.

$(3) \Rightarrow(1)$. This follows immediately from (6.5).

REMARK. Suppose that $B$ has an order unit $e$ such that $\|e\|=1$ and its norm is of the form

$$
\|a\|=\inf \{\lambda>0:-\lambda e \leq a \leq \lambda e\} .
$$

Then

$$
N(a)=\inf \{\lambda>0: a \leq \lambda e\}
$$

and $B$ is of type $(N, \infty)$. For every $a \in B$,

$$
a=\frac{1}{2}(\|a\| e+a)-\frac{1}{2}(\|a\| e-a),
$$

and, hence, $B_{1}$ is positively generated. Therefore $B^{*}$ is of type $(N, 1)$ and the Jordan decompositions are $N$-decompositions. Since $B_{1}$ is positively generated, $B\left(\mu_{\infty}\right)=B_{1} \subset P-P$. However $B\left(\mu_{\infty}\right)=B\left(\mu_{1}^{\#}\right)=\overline{P-P}$. Therefore $P-P$ is closed. Note that the decomposition of $a$,

$$
a_{+}=\frac{1}{2}(\|a\| e+a) \text { and } a_{-}=\frac{1}{2}(\|a\| e-a) \text {, }
$$

is an orthogonal decomposition and

$$
\|a\|=N(a) \vee N(-a)=\left\|a_{+}+a_{-}\right\|=\left\|a_{+}\right\| \vee\left\|a_{-}\right\| \text {. }
$$

However these are not enough to conclude that $\left(a_{+}, a_{-}\right)$is an $N$-decomposition of $a$. Note also that the equality

$$
a=N(a) e-(N(a) e-a)
$$

shows that $B$ is $N_{+}$-decomposable and, hence, $P-B_{+}$is closed.

\section{The Robinson property and spaces of type $(N, p)$}

The connection between the Robinson property and the spaces of type $(N, p)$ is based on the following fact, which is a generalized version of (3.5).

(7.1). Suppose that every $f \in B^{*}$ admits a decomposition $f=f_{+}-f_{-}$such that $f_{ \pm} \geq 0$ and

$$
\left\|f_{+}\right\| N(a)+\left\|f_{-}\right\| N(-a) \leq\|f\|\|a\| \text { for azz } a \in B \text {. }
$$


Then $\bar{N}(u)=\|u\|$ for all $u \in L(B)_{+}$if and only if the dual norm is monotone.

Proof. Let $u \in L(B)_{+}, a \in B_{1}$ and $f \in B_{1}^{*}$. Then

$$
\begin{aligned}
f(u(a)) & =f_{+}(u(a))+f_{-}(u(-a)) \\
& \leq\left\|f_{+}\right\| N(u(a))+\left\|f_{-}\right\| N(u(-a)) .
\end{aligned}
$$

Suppose that the dual norm is monotone. Then there exist $b_{n} \geq 0$ and $c_{n} \geq 0$ such that

$$
b_{n} \geq a, \quad c_{n} \geq-a, \quad\left\|b_{n}\right\| \leq N(a)+1 / n \text { and }\left\|c_{n}\right\| \leq N(-a)+1 / n \text {. }
$$

Then, since $u \geq 0$,

$$
\begin{aligned}
f(u(a)) & \leq\left\|f_{+}\right\| N\left(u\left(b_{n}\right)\right)+\left\|f_{-}\right\| N\left(u\left(c_{n}\right)\right) \\
& \leq\left\|f_{+}\right\| \bar{N}(u)\left\|b_{n}\right\|+\left\|f_{-}\right\| \bar{N}(u)\left\|c_{n}\right\| \\
& \leq \bar{N}(u)\left(\left\|f_{+}\right\|(N(a)-(z / n))+\left\|f_{-}\right\|(N(-a)-(1 / n))\right) .
\end{aligned}
$$

Therefore we have $\|u\| \leq \bar{N}(u)$ and, hence, $\|u\|=\bar{N}(u)$. Conversely, if $\|u\|=\bar{N}(u)$ for all $u \in L(B)_{+}$, the norm of $L(B)$ is monotone. Therefore the dual norm is monotone.

As a corollary, we have a sufficient condition for the Robinson property.

(7.2). Suppose that $B$ is of type $(N, p)$ and $B^{*}$ is $N$-decomposable. Then $B$ has the Robinson property.

Proof. By (6.5) the dual norm is of type $(N, q)$ and, for an $N$-decomposition $f=f_{+}-f_{-}$of $f \in B^{*}$, we have

$$
\begin{aligned}
\left\|f_{+}\right\| N(a)+\left\|f_{-}\right\| N(-a)=N(f) & N(a)+N(-f) N(-a) \\
& \leq\left(N(f)^{q}+N(-f)^{q}\right)^{1 / q}\left(N(a)^{p}+N(-a)^{p}\right)^{1 / p}=\|f\|\|a\| .
\end{aligned}
$$

Furthermore, since the norm of $B$ is monotone, we have $\bar{N}(u)=\|u\|_{+}$for $u \in L(B)_{+}$. Therefore $B$ has the Robinson property.

It follows from the remark at the end of the previous section that the space whose norm is an order unit norm has the Robinson property. 


\section{References}

[1] Wolfgang Arendt, Paul R. Chernoff and Tosio Kato, "A generalization of dissipativity and positive semigroups", J. Operator Theory 8 (1982), 167-180.

[2] J. Grosberg et M. Krein, "Sur la décomposition des fonctionelles en composantes positives", C.R. (DokZ.) Acad. Sci. URSS (N.S.) 25 (1939), 723-726.

[3] Л.В. Канторович, Б.З. Вулих и А.Г. Пинскер [L.V. Kantorovitch, В.z. Vulikh and A.G. Pinsker], Функщоналъный анализ в полуупорядоченнах пространствнх [Functional analysts in partially ordered spaces ] (Gos. Izdat. Tehn.-Teor. Lit., Moscow, 1950).

[4] Derek W. Robinson, "Continuous semigroups on ordered Banach spaces" (Mathematics Research Report, 17. Australian National University, Canberra, 1982).

[5] Derek W. Robinson, "On positive semigroups" (Mathematics Research Report, 18. Australian National University, Canberra, 1982).

[6] Derek W. Robinson and Sadayuki Yamamuro, "The Jordan decomposition and half-norms", Pacific J. Math. (to appear).

[7] Derek W. Robinson and Sadayuki Yamamuro, "Addition of an identity to an ordered Banach space", J. Austral. Math. Soc. Ser. A (to appear).

[8] Derek W. Robinson and Sadayuki Yamamuro, "Hereditary cones, order ideals and half-norms", Pacific J. Math. (to appear).

[9] Derek W. Robinson and Sadayuki Yamamuro, "The canonical half-norm, dual half-norms and monotonic norms", Töhoku Math. J. (to appear).

Department of Mathematics, Institute of Advanced Studies, Australian National University, PO Box 4, Canberra, ACT 2600, Australia. 